\title{
Functional analysis of granulocyte and monocyte subpopulations in neonates
}

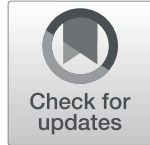

Ines Hegge ${ }^{1 \dagger}$, Ferry Niepel ${ }^{1 \dagger}$, Anja Lange ${ }^{1}$, Antje Vogelgesang ${ }^{2}$, Matthias Heckmann ${ }^{1}$ and Johanna Ruhnau ${ }^{2^{*}}$ (D)

\begin{abstract}
Background: Neonate immune cell functions lack full protection against pathogens. This could be either defect or protective mechanism against overshooting proinflammatory immune responses.

We here analysed the function of classical, pro- and anti-inflammatory monocytes and granulocytes from neonates in comparison with adults to investigate if suppressed functions of subpopulations are causative for the unique neonatal immune status. Therefore, reactive oxygen species (ROS) and surface activation markers were quantified in subpopulations.

Methods: In a prospective, longitudinal study granulocyte and monocyte subpopulations were analysed in healthy term infants (> 37 week; $n=13$ ) in comparison with healthy young adults $(n=11)$. Percentage (\%) of cells expressing surface marker (HLA-DR, CD11b, CD62L, CD32, Toll-Like-Receptor-2) and expression per cell, determined by mean fluorescence intensity (MFI), were measured by flow cytometry. ROS production was induced by fMLP, PMA and E. coli in term neonates (> 37 week; $n=13$ ).
\end{abstract}

Results: Classical granulocytes were down- and proinflammatory granulocytes upregulated in neonates compared with adults. Percentage of TLR-2 expressing granulocytes was increased in neonates. Granulocytic ROS production depended on stimulation. The percentage of anti-inflammatory monocytes was increased, while classical monocytes were reduced in neonates. HLA-DR (\%, MFI) showed reduction for all monocyte subpopulations, while CD32, CD1 1b, CD62L and TLR-2 were differently regulated in comparison with adults.

Conclusions: Differentially regulated granulocyte and monocyte subpopulations indicate a unique state of neonatal immunity to fight infections and prevent dysregulation. Further studies are needed to investigate the role of reduced granulocytic ROS formation and reduced monocytic HLA-DR in active disease.

Keywords: Neonate, Subpopulations, Innate immunity, ROS

\section{Background}

As the adaptive immunity of newborns is mostly naive and immune responses will take longer to develop as compared with adults, the innate immune response, mainly carried out by neutrophils and monocytes, is of major importance for early-life pathogen defence [1]. But newborn neutrophils show characteristics of impaired bacterial defence function like reduced expression of Tolllike receptor (TLR) 2 [2] and reduced formation of neutrophil extracellular traps [3] -important mechanisms for recognition and trapping of pathogens. Furthermore, monocytes express less HLA-DR which is known to be a

\footnotetext{
* Correspondence: johanna.ruhnau@uni-greifswald.de

${ }^{\dagger}$ Ines Hegge and Ferry Niepel contributed equally to this work.

${ }^{2}$ Department of Neurology, University Medicine Greifswald, F.-Sauerbruch-Str.,

17475 Greifswald, Germany

Full list of author information is available at the end of the article
}

marker of immunosuppression and a predictor of sepsis development in neonates [4]. Monocytes are also impaired in uptake of bacteria by phagocytosis [5].

Recently Brook et al. stated that the partly lowered antibacterial function of innate immune cells like neutrophils and monocytes is rather a mechanism of protection from overshooting proinflammatory immune responses than a pathologic defect [6]. A broad range of pro-inflammatory plasma proteins is upregulated in septic neonates in comparison with healthy controls, indicating an overwhelming and consuming proinflammatory immune response against infection in early life [7].

Granulocytes have been differentiated in three different main subpopulations by CD16, a Fc-receptor, and CD62L, a L-Selectin which is important for cell adhesion [8-10]. The largest granulocyte subset within the 
peripheral blood, referred to as "classical granulocytes," expresses high levels of $\mathrm{CD} 16$ and to some degree CD62L $\left(\mathrm{CD} 16^{+} \mathrm{CD} 62 \mathrm{~L}^{+}\right)$, proinflammatory granulocytes are defined as $\mathrm{CD} 16^{\mathrm{dim}} \mathrm{CD} 62 \mathrm{~L}^{+}$and anti-inflammatory granulocytes as $\mathrm{CD}{ }^{+} \mathrm{CD} 6 \mathrm{~L}^{-}$. To date, no data about the different regulation of granulocyte subpopulations in neonates exist. Similar to granulocytes, monocytes can be distinguished in three different subpopulations in regard to their expression of CD14 and CD16: (i) classical monocytes $\left(\mathrm{CD} 14^{+} \mathrm{CD} 16^{-}\right)$; (ii) anti-inflammatory $\left(\mathrm{CD} 14^{+} \mathrm{CD} 16^{+}\right)$; and (iii) proinflammatory monocytes $\left(\mathrm{CD} 14^{\mathrm{dim}} \mathrm{CD} 16^{+}\right)$.

An altered composition of granulocyte or monocyte subsets might be part of the unique immune system configuration after birth which prevents accelerating immune responses. Therefore, we analysed granulocytes and monocytes in term neonates to gain a better understanding of the regulation of their subpopulations in comparison with young adults. This knowledge will allow future studies to investigate the role of these subpopulations for increased infection rates in term or preterm neonates.

The Fc-receptor CD32 mediates multiple cell-type specific functions including phagocytosis or release of inflammatory mediators, which are important functions of innate immune cells [11]. CD11b plays a critical role in pathogen recognition. Its downstream signalling initiates immune responses that ultimately link to the generation of adaptive immunity [12]. Since alterations of activation markers could be a part of the optimal balance between immune resistance and response TLR-2, HLA-DR, CD32, CD11b and CD62L were analysed on monocyte and granulocyte subpopulations.

The production of reactive oxygen species (ROS) is one of the best analysed anti-bacterial mechanisms of neonatal neutrophils. Nevertheless, it is partly discussed controversially [13, 14]. ROS production within respiratory burst of neonates was reported to be as effective as the one of adults [15]. In contrast stressed neonatal neutrophils showed a significantly elevated response when compared with those from adult controls [16]. Since these data lack an analysis of granulocyte subpopulations we here analysed the three subpopulations in regard to their production of ROS as determined by the conversion of dihydrorhodamine (DHR) into rhodamine.

\section{Methods}

\section{Study population}

Healthy term neonates were recruited between July 2016 to March 2017 and May 2018 to September 2018 at the University Medicine Greifswald. Healthy young adults served as controls.

Monocyte and granulocyte subpopulations were analysed in 11 healthy term neonates (mean gestational age $=39$ weeks +2 days (SD, 1 weeks +2 days); male $=7$, female $=4$; birth mode: spontaneous $=1$; primary caesarean section $=9$; secondary caesarean section $=1$ ) and 10 young healthy adults ( mean age $=23.5$ years $(\mathrm{SD}=4.9$ years), male $=2$, female $=8$ ).

For ROS production experiments samples of 13 healthy term neonates (mean gestational age, 38 weeks +4 days $(\mathrm{SD}, 0$ weeks +6 days); male $=5$, female $=8$; birth mode: spontaneous $=5$; primary caesarean section $=8$; secondary caesarean section $=0$ ) and 11 young healthy adults (mean age, 25 years (SD, 6.4 years); male = 5 , female $=6$ ) were used.

Exclusion criteria were severe congenital malformations, chromosomal aberrations, perinatal infection or chorioamnionitis and lack of written consent (see Additional file 1: Table S1 for details).

\section{Monocyte and granulocyte subtypes}

EDTA cord blood was sampled at birth and processed within $2 \mathrm{~h}$ to examine neonatal monocytes and granulocytes. After a red blood cell lysis using ACK lysing buffer (155 $\mathrm{mM} \mathrm{NH}_{4} \mathrm{Cl}, 10 \mathrm{mM} \mathrm{KHCO} 3,0.1 \mathrm{mM}$ EDTA) to induce cell swelling and rupture of membranes, cells were stained by Zombie NIR ${ }^{\mathrm{Tw}}$ Fixable Viability Kit (BioLegend $^{\mathrm{Tm}}$ ) on ice for $15 \mathrm{~min}$ to distinguish dead and alive cells, followed by a second staining with the different cell surface antibodies for $10 \mathrm{~min}$ on ice. Cells were fixed in $1 \%$ paraformaldehyde (PFA). Immune cell subpopulations of monocytes and granulocytes were analysed by flow cytometry (BD LSR II) as defined by anti-HLA-DR Alexa Flour 488, anti-CD11b Brilliant Violet 421, anti-CD14 PerCP/Cy5.5, anti-CD16 Brilliant Violet 650, anti-CD62L PE-Cy7, anti-TLR-2 Alexa 647 and anti-CD32-PE (BioLegend). The results were evaluated using FlowJo Software 7.6.5 (Tree Star Inc., Ashland, OR, USA). Results are shown as percentages of granulocytes, monocytes or their subpopulations. To display the expression of activation markers, mean fluorescence intensity (MFI) was used. For the differentiation of monocytes and granulocyte subpopulation as well as activation marker, fluorescence minus one controls (FMO) were used. CD14 $4^{\mathrm{dim}}$ monocyte and CD16 ${ }^{\text {dim }}$ neutrophil population was distinguished by gating the 25th percentile of main monocyte and neutrophil population, respectively (Additional file 2: Figure S1A/B).

\section{ROS production}

ROS production was quantified by flow cytometry using the Phagoburst Kit (Glycotope Biotechnology GmbH) according to manufacturer's instructions. Briefly, heparinized cord blood was incubated on ice with anti-CD14 APC/Cy7, anti-CD16 APC and anti-CD62L Brilliant Violet 421 antibodies (Biolegend). For detailed gating strategy see Additional file 3: Figure S2. Cells either remained unstimulated or were incubated with unlabeled opsonized E. coli $\left(0.9-1.8 \times 10^{8} / \mathrm{ml}\right)$, phorbol 12 myristate 13-acetate (PMA) $(0.74 \mu \mathrm{M})$, or $\mathrm{N}$-formyl- 
methionyl-leucyl-phenylalanine (fMLP) $(0.45 \mu \mathrm{M})$ as stimulants for $10 \mathrm{~min}$ at $37^{\circ} \mathrm{C}$; subsequently, DHR was added for $10 \mathrm{~min}$ which-by ROS-dependent conversion into rhodamine 123-allowed the quantification of reactive oxidants and determination of the percentage of phagocytes that produced ROS. The ROS production per cell was quantified by MFI. Kit-included DNA-Dye was used after red blood cell lysis using PFA-containing BD FACS ${ }^{\mathrm{Tx}}$ Lysing Solution to differentiate between E. coli and cells. The flow cytometry results were evaluated with Flowjo Software 10.3 (Tree Star Inc., Ashland, OR). Gating of subpopulation was done as described above (Additional file 2: Figure S1 A/B; Additional file 3: Figure S2).

\section{Statistical analysis}

All data sets were tested for adherence to the Gaussian distribution with the Kolmogorov-Smirnov test. Since some of the data failed the normality test we used nonparametric testing throughout. The Kruskal-Wallis test or Friedman test with Dunn's multiple comparison test as a post-test or the Mann-Whitney test were used as appropriate. GraphPad-PRISM 5.0 (GraphPad Software Inc., San Diego, CA, USA) was used for all analyses. A $p$ value $\leq 0.05$ was regarded as significant.

\section{Results}

\section{Differently regulated granulocyte subpopulations}

Compared with adult controls neonates showed a reduced percentage of classical granulocytes $\mathrm{CD} 16^{+} \mathrm{CD} 62 \mathrm{~L}^{+}$cells while proinflammatory $\mathrm{CD} 16{ }^{\mathrm{dim}} \mathrm{CD} 62 \mathrm{~L}^{+}$cells were increased. Anti-inflammatory $\mathrm{CD}_{16}{ }^{+} \mathrm{CD} 62 \mathrm{~L}^{-}$cells were not altered in comparison with controls (Fig. 1a-d).

\section{Activation marker of granulocyte and their subpopulations}

To analyse the activation profile of granulocytes and their subpopulations in comparison with controls, we analysed the percentage of CD11b, CD32 and TLR-2 expressing cells as well as the amount of activation marker per cell by MFI.

Reduced percentages of $\mathrm{CD} 11 \mathrm{~b}$ positive granulocytes and CD32 positive granulocytes were detected in neonates in comparison with controls. Neonates showed a higher percentage of TLR-2 on all granulocyte subpopulations compared with controls (Table 1). The amount of TLR-2 expression on proinflammatory $\mathrm{CD} 16{ }^{\mathrm{dim}} \mathrm{CD} 62 \mathrm{~L}^{+}$granulocytes was decreased in neonatal cord blood (Fig. 2).

\section{ROS production of granulocyte subpopulations}

Percentage of ROS-producing granulocyte subpopulation and ROS amount per cell (MFI) was quantified (Fig. 3). Neonates showed enhanced ROS amount per cell for classical $\mathrm{CD}_{16}{ }^{+} \mathrm{CD} 62 \mathrm{~L}^{+}$and anti-inflammatory $\mathrm{CD} 16^{+} \mathrm{CD} 62 \mathrm{~L}^{-}$subpopulation in unstimulated and fMLP-stimulated cells. In addition, ROS amount per cell was upregulated in proinflammatory $\mathrm{CD} 16{ }^{\mathrm{dim}} \mathrm{CD} 62 \mathrm{~L}^{+}$cells after PMA stimulation. In $E$. coli-stimulated samples all neonatal granulocytes as well as their subpopulations showed a higher ROS production per

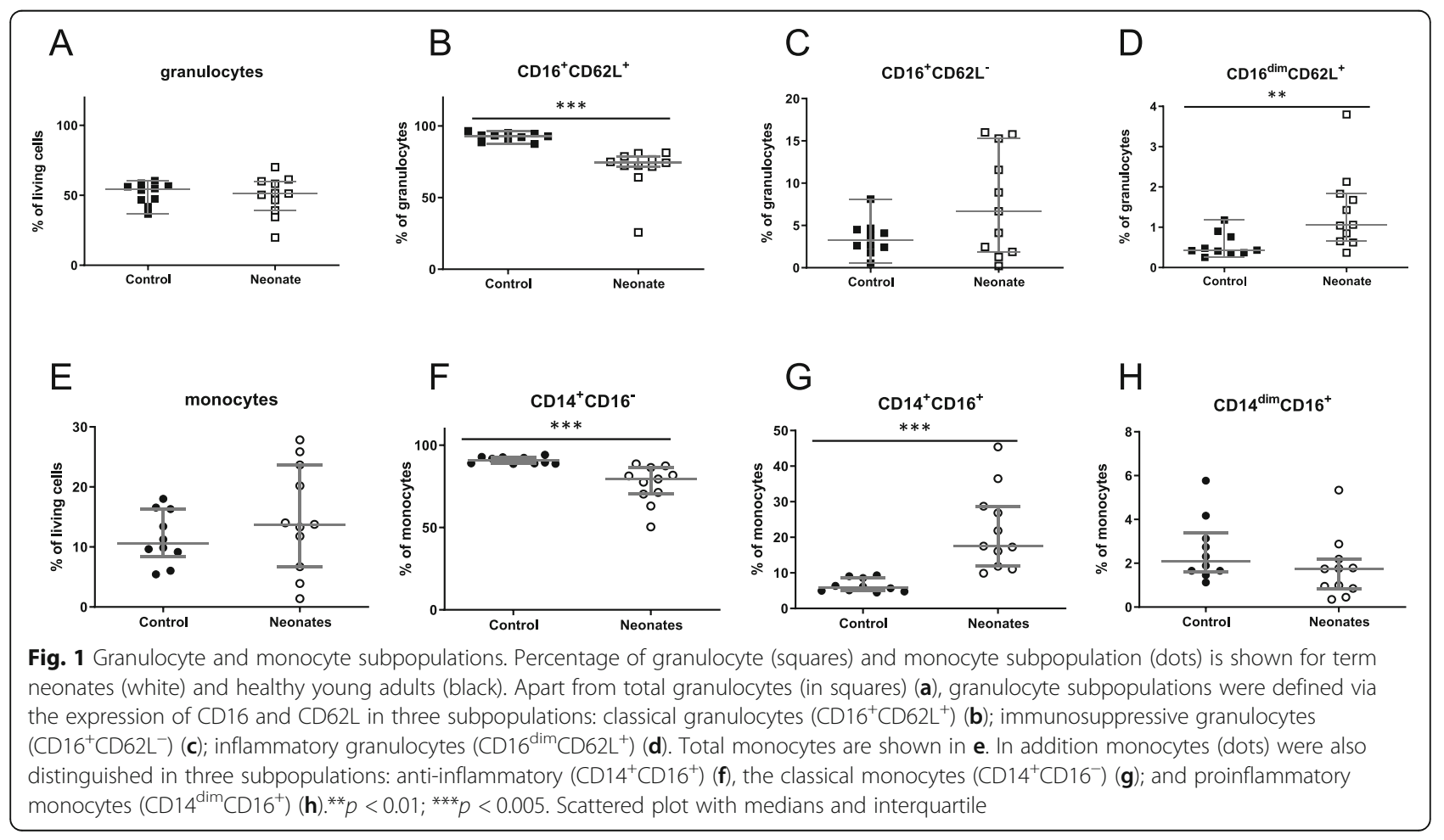


Table 1 Summary of all observed marker on granulocyte and monocytes and their subpopulations

\begin{tabular}{|c|c|c|c|c|c|}
\hline & Marker & Change (compared with adult) & Adult (median (Min-Max)) & Neonate (median (Min-Max)) & $p$ value (Mann-Whitney test) \\
\hline \multirow[t]{10}{*}{ Granulocytes } & $C D 11 b+(\%)$ & $\downarrow$ & 98.94 (97.83-99.78) & $95.27(29.87-97.73)$ & 0.0001 \\
\hline & $C D 11 b+(M F I)$ & - & $3423(2274-6123)$ & $2936(1847-5151)$ & 0.5974 \\
\hline & CD16+ (\%) & $\downarrow$ & 98.05 (93.91-99.32) & 89.59 (28.21-96.12) & 0.0002 \\
\hline & CD16+ (MFI) & $\downarrow$ & $58.687(40.735-76.909)$ & $26.939(26.309-36.276)$ & 0.0001 \\
\hline & CD32+ (\%) & $\downarrow$ & 99.53 (98.11-99.85) & $97.14(32.18-99.41)$ & 0.0017 \\
\hline & CD32+ (MFI) & - & $16.466(12.504-27.052)$ & $19.779(11.886-24.551)$ & 0.3418 \\
\hline & CD62L+ (\%) & $\downarrow$ & 96.55 (93.15-99.34) & 85.07 (32.05-92.36) & 0.0001 \\
\hline & CD62L+ (MFI) & $\downarrow$ & $43.841(21.105-64.313)$ & $19.172(12.638-31.370)$ & 0.0028 \\
\hline & TLR2+ (\%) & $\uparrow$ & $2.9(0.6-10.98)$ & $8.36(3.32-72.73)$ & 0.0035 \\
\hline & TLR2+ (MFI) & - & $1506(190.9-5427)$ & 778.7 (365.3-3335) & 0.8053 \\
\hline \multirow[t]{3}{*}{$\begin{array}{l}\text { Granulocytes } \\
\text { subsets }\end{array}$} & $\begin{array}{l}\text { CD16+ } \\
\text { CD62L+ }\end{array}$ & $\downarrow$ & 93.01 (87.46-96.55) & 74.37 (25.78-81.35) & 0.0001 \\
\hline & $\begin{array}{l}\text { CD16+ } \\
\text { CD62L- }\end{array}$ & - & $3.24(0.54-8.1)$ & $6.68(0.21-16.0)$ & 0.2453 \\
\hline & $\begin{array}{l}\text { CD16dim } \\
\text { CD62L+ }\end{array}$ & $\uparrow$ & $0.425(0.25-1.18)$ & $1.06(0.37-3.8)$ & 0.0092 \\
\hline \multirow{6}{*}{$\begin{array}{l}\text { CD16+CD62L+ } \\
\text { (classical) }\end{array}$} & $C D 11 b+(\%)$ & - & $100.0(98.67-100)$ & $99.40(97.76-100)$ & 0.0545 \\
\hline & CD11b+ (MFI) & - & 3439 (2284-6139) & $2978(1880-5259)$ & 0.5974 \\
\hline & CD32+ (\%) & - & $100.0(100.0-100.0)$ & $100.0(99.97-100.0)$ & - \\
\hline & CD32+ (MFI) & - & 16.655 (12.794-27.373) & $20.943(13.267-27.917)$ & 0.098 \\
\hline & TLR2+ (\%) & $\uparrow$ & $0.985(0.32-11.52)$ & $5.75(0.46-79.95)$ & 0.0346 \\
\hline & TLR2+ (MFI) & - & $1598(360.3-5217)$ & $1013(613.4-3321)$ & 0.8603 \\
\hline \multirow{6}{*}{$\begin{array}{l}\text { CD16+CD62L- } \\
\text { (anti-inflammatory) }\end{array}$} & $C D 11 b+(\%)$ & - & 98.43 (94.62-99.85) & 98.17 (92.92-99.48) & 0.3787 \\
\hline & CD11b+ (MFI) & - & 3332 (2040-5834) & $2753(1686-3902)$ & 0.3418 \\
\hline & CD32+ (\%) & - & 99.99 (99.61-100.0) & $99.96(54.96-100.0)$ & 0.0587 \\
\hline & CD32+ (MFI) & - & $15.244(11.030-26.700)$ & $19.774(13.674-27.184)$ & 0.0726 \\
\hline & TLR2+ (\%) & $\uparrow$ & $0.98(0.27-6.67)$ & $5.97(0.57-81.36)$ & 0.0183 \\
\hline & TLR2+ (MFI) & - & $2028(443.6-7026)$ & $941.5(634.4-3216)$ & 0.4181 \\
\hline \multirow{6}{*}{$\begin{array}{l}\text { CD16dimCD62L+ } \\
\text { (pro-inflammatory) }\end{array}$} & $C D 11 b+(\%)$ & - & $98.46(81.77-100)$ & 97.05 (82.95-99.86) & 0.5495 \\
\hline & CD11b+ (MFI) & - & $2457(1516-5741)$ & $2731(2171-4653)$ & 0.3787 \\
\hline & CD32+ (\%) & - & $100(98.5-100.0)$ & 99.97 (99.36-100.0) & 0.9697 \\
\hline & CD32+ (MFI) & - & $8236(5792-16.004)$ & 7168 (4230-10.209) & 0.5035 \\
\hline & TLR2+ (\%) & $\uparrow$ & $1.4(0.0-5.5)$ & $9.18(0.73-35.08)$ & 0.0043 \\
\hline & TLR2+ (MFI) & $\downarrow$ & $2625(0.00-5333)$ & $657.8(446.7-3439)$ & 0.0378 \\
\hline \multirow[t]{12}{*}{ Monocytes } & CD11b+ (\%) & $\downarrow$ & 99.51 (98.50-99.96) & 98.64 (95.76-99.33) & 0.0137 \\
\hline & CD11b+ (MFI) & - & $2694(1256-6598)$ & 2093 (993.4-4453) & 0.1489 \\
\hline & CD16+ (\%) & $\uparrow$ & $9.33(5.91-11.38)$ & 21.26 (10.97-49.66) & 0.0003 \\
\hline & CD16+ (MFI) & $\downarrow$ & $12.126(5745-19.915)$ & $3684(1817-5825)$ & 0.0002 \\
\hline & CD32+ (\%) & - & 99.93 (99.04-99.98) & 99.65 (92.43-99.82) & 0.0724 \\
\hline & CD32+ (MFI) & - & $22.733(12.835-31.849)$ & 19.335 (13.609-30.177) & 0.5035 \\
\hline & CD62L (\%) & $\downarrow$ & 94.24 (55.77-96.31) & 80.15 (27.67-96.15) & 0.0083 \\
\hline & CD62L+ (MFI) & $\downarrow$ & $28.574(15.121-61.757)$ & $11.974(5130-25.963)$ & 0.0011 \\
\hline & HLA-DR+ (\%) & $\downarrow$ & 99.51 (97.14-99.83) & 94.46 (85.72-97.56) & 0.0002 \\
\hline & $\begin{array}{l}\text { HLA-DR+ } \\
\text { (MFI) }\end{array}$ & $\downarrow$ & $5050(3839-5678)$ & $2008(1135-4030)$ & 0.0002 \\
\hline & TLR2+ (\%) & $\downarrow$ & 99.07 (95.67-99.83) & 97.27 (92.02-99.30) & 0.0317 \\
\hline & TLR2+ (MFI) & - & $3693(2511-5553)$ & $3166(1271-5754)$ & 0.1131 \\
\hline
\end{tabular}


Table 1 Summary of all observed marker on granulocyte and monocytes and their subpopulations (Continued)

\begin{tabular}{|c|c|c|c|c|c|}
\hline & Marker & Change (compared with adult) & Adult (median (Min-Max)) & Neonate (median (Min-Max)) & $p$ value (Mann-Whitney test) \\
\hline \multirow[t]{3}{*}{ Monocyte subsets } & $\begin{array}{l}\text { CD14+ } \\
\text { CD16+ }\end{array}$ & $\uparrow$ & $5.925(4.47-9.33)$ & $17.52(9.89-45.38)$ & 0.0001 \\
\hline & CD14+CD16- & $\downarrow$ & 90.59 (88.62-94.09) & $79.4(50.34-88.55)$ & 0.0001 \\
\hline & $\begin{array}{l}\text { CD14dim } \\
\text { CD16+ }\end{array}$ & - & $2.1(1.12-5.77)$ & $1.74(0.35-5.34)$ & 0.1299 \\
\hline \multirow{10}{*}{$\begin{array}{l}\text { CD14+CD16- } \\
\text { (classical) }\end{array}$} & $C D 11 b+(\%)$ & $\downarrow$ & 99.77 (98.59-99.98) & 98.26 (94.89-99.29) & 0.0006 \\
\hline & $\mathrm{CD} 11 \mathrm{~b}+(\mathrm{MFI})$ & - & $2709(1250-6706)$ & 2025 (914.2-4189) & 0.13 \\
\hline & CD32+ (\%) & $\downarrow$ & $99.94(99.27-99.99)$ & $99.67(93.55-99.8)$ & 0.0183 \\
\hline & CD32+ (MFI) & - & $22.650(12.310-31.390)$ & $18.040(12.880-27.480)$ & 0.2453 \\
\hline & CD62L+ (\%) & $\downarrow$ & $96.81(57.66-98.74)$ & $82.53(26.2-98.1)$ & 0.0054 \\
\hline & CD62L+ (MFI) & $\downarrow$ & $29.690(15.240-64.070)$ & $12.150(5195-28.160)$ & 0.0014 \\
\hline & HLA-DR+ (\%) & $\downarrow$ & 99.57 (98.29-99.87) & 95.59 (88.84-99.24) & 0.0003 \\
\hline & $\begin{array}{l}\text { HLA-DR+ } \\
\text { (MFI) }\end{array}$ & $\downarrow$ & $4260(3050-4736)$ & $1833(907.4-2658)$ & 0.0001 \\
\hline & TLR2+ (\%) & $\downarrow$ & 99.69 (97.23-99.96) & 97.89 (94.51-99.29) & 0.0151 \\
\hline & TLR2+ (MFI) & - & $3671(2465-5485)$ & $2944(1225-5206)$ & 0.0845 \\
\hline \multirow{10}{*}{$\begin{array}{l}\text { CD14+CD16+ (anti- } \\
\text { inflammatory) }\end{array}$} & $C D 11 b+(\%)$ & - & 99.85 (97.72-100.0) & 99.92 (98.06-100.0) & 0.5714 \\
\hline & CD11b+ (MFI) & - & $3009(1441-6496)$ & $2643(1357-5016)$ & 0.4181 \\
\hline & CD32+ (\%) & - & $99.93(96.42-100.0)$ & 99.77 (90.23-99.94) & 0.4165 \\
\hline & CD32+ (MFI) & - & $24.030(14.400-40.560)$ & $22.930(16.130-34.550)$ & 0.6985 \\
\hline & CD62L+ (\%) & - & 74.74 (35.97-86.96) & 75.91 (42.13-95.42) & 0.5973 \\
\hline & CD62L+ (MFI) & $\downarrow$ & $16.240(11.660-23.330)$ & $12.150(4641-20.090)$ & 0.0221 \\
\hline & HLA-DR+ (\%) & $\downarrow$ & $99.16(87.21-100.0)$ & 93.53 (64.45-96.81) & 0.0378 \\
\hline & $\begin{array}{l}\text { HLA-DR+ } \\
\text { (MFI) }\end{array}$ & $\downarrow$ & $14.880(10.390-19.610)$ & $3219(2243-7424)$ & 0.0001 \\
\hline & TLR2+ (\%) & - & 98.54 (82.51-99.77) & 95.53 (87.48-99.86) & 0.5495 \\
\hline & TLR2+ (MFI) & - & $4893(3280-6613)$ & $3546(1492-6474)$ & 0.0528 \\
\hline \multirow{10}{*}{$\begin{array}{l}\text { CD14 }{ }^{\operatorname{dim}} \text { CD } 16+ \\
\text { (pro-inflammatory) }\end{array}$} & CD11b+ (\%) & - & $98.17(89.87-99.83)$ & $96.82(86.89-98.53)$ & 0.3418 \\
\hline & CD11b+ (MFI) & - & $1523(803.7-3416)$ & $1250(1027-2207)$ & 0.5974 \\
\hline & CD32+ (\%) & - & $98.87(85.65-100.0)$ & $96.11(83.81-100.0)$ & 0.09 \\
\hline & CD32+ (MFI) & - & $15.860(10.070-34.790)$ & $14.650(11.360-22.380)$ & 0.2178 \\
\hline & CD62L+ (\%) & - & $32.15(13.08-44.67)$ & $34.89(5.3-53.33)$ & 0.8053 \\
\hline & CD62L+ (MFI) & - & $9512(4503-31.870)$ & $16.650(7670-25.480)$ & 0.1131 \\
\hline & HLA-DR+ (\%) & $\downarrow$ & 92.48 (75.53-98.71) & 63.51 (46.67-95.24) & 0.0151 \\
\hline & $\begin{array}{l}\text { HLA-DR+ } \\
\text { (MFI) }\end{array}$ & $\downarrow$ & $12.550(7028-15.170)$ & 8144 (3757-11.960) & 0.0017 \\
\hline & TLR2+ (\%) & - & 84.39 (61.21-97.33) & 76.67 (41.11-91.01) & 0.1697 \\
\hline & TLR2+ (MFI) & - & 3529 (2709-5068) & $3104(1390-4592)$ & 0.13 \\
\hline
\end{tabular}

cell in comparison with controls. A lower percentage of ROS-producing proinflammatory CD16 ${ }^{\text {dim }} \mathrm{CD} 62 \mathrm{~L}^{+}$cells was measured for unstimulated and fMLP-stimulated cells in neonates. PMA stimulation induced a reduced percentage of ROS-producing anti-inflammatory $\mathrm{CD}_{16}{ }^{+} \mathrm{CD} 62 \mathrm{~L}^{-}$. An explorative subanalysis of sex showed no significant differences ( $p \geq 0.9999$; data not shown).

\section{Differently regulated monocyte subpopulations}

Monocyte subpopulations were differentially regulated in neonates compared with controls. Classical $\mathrm{CD} 14^{+} \mathrm{CD} 16^{+}$cells were enhanced in neonatal cord blood in comparison with lower number of antiinflammatory $\mathrm{CD} 14^{+} \mathrm{CD} 16^{-}$cells and unaltered proinflammatory CD $14^{\text {dim }}{ }^{\text {CD } 16^{+}}$cells (Fig. 1e-h). 

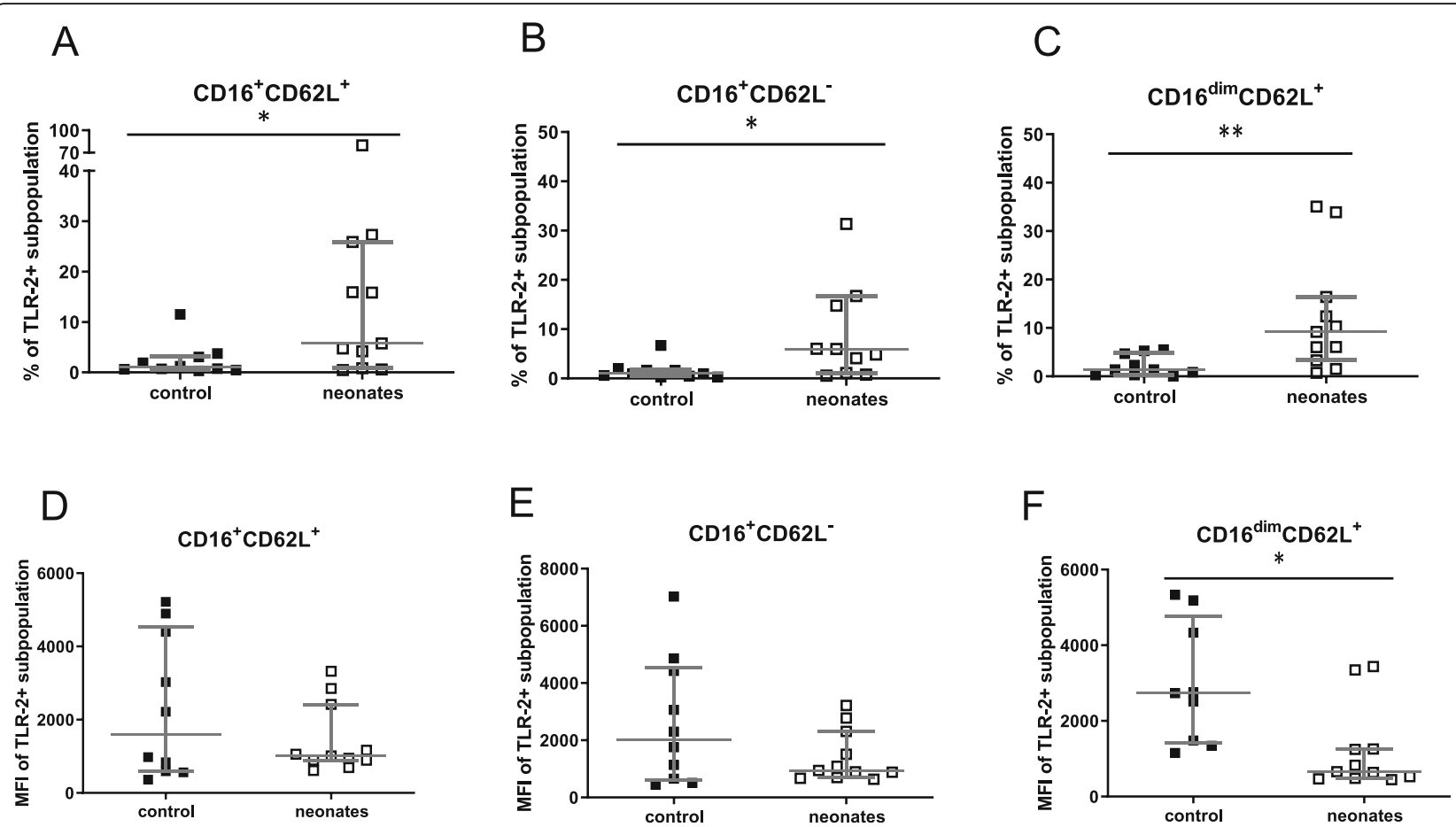

Fig. 2 TLR-2 as activation marker on granulocytes and granulocyte subpopulations. Neonates (white squares) were compared with healthy controls (black squares). Percentage (\%) (a-c) TLR-2 on the surface of cells and expression of TLR-2 as mean fluorescence intensity (MFI) (D-F) is shown for different granulocyte subset. In (a) and (d) classical granulocytes $\left(\mathrm{CD} 16^{+} \mathrm{CD} 62 \mathrm{~L}^{+}\right)$; in (b) and (e) immunosuppressive granulocytes $\left(\mathrm{CD} 16^{+} \mathrm{CD} 62 \mathrm{~L}^{-}\right)$and in (c) and (f) inflammatory granulocytes $\left(\mathrm{CD} 16^{\mathrm{dim}} \mathrm{CD} 62 \mathrm{~L}^{+}\right)$are shown. ${ }^{*} p<0.05 ;{ }^{* *} p<0.01$. Scattered plot with medians and interquartile

Activation marker of monocytes and their subpopulations To analyse the activation profile of monocytes in comparison with healthy controls, percentages of HLA-DR, CD11b, CD62L and TLR-2 bearing cells as well as the amount per cell (MFI) were analysed.

Percentage of CD11b, CD62L, HLA-DR and TLR-2 bearing monocytes was reduced in neonatal compared with adult monocytes. The amount of CD62L and HLA-DR was significantly decreased on neonatal monocytes (Table 1).

Percentages of CD32, TLR-2 and CD11b positive classical CD $14^{+} \mathrm{CD} 16^{-}$monocytes were decreased in neonates compared with controls. Neonatal $\mathrm{CD} 14^{+} \mathrm{CD} 16^{-}$monocytes showed a significant decrease in CD62L positive cells and CD62L amount on cell surface. In anti-inflammatory $\mathrm{CD} 14^{+} \mathrm{CD} 16^{+}$cells CD62L amount was reduced (Table 1 ). The decrease of HLA-DR positive cells in neonates as well as the decrease of HLA-DR amount was measured for all three monocyte subpopulations (Fig. 4).

\section{Discussion}

The function of innate immune cells in neonates is known to be different from adults. These immune alterations might be part of a balanced system between prevention of hyperinflammation and defence of pathogens [6]. Therefore, the regulation also of innate immune cells subpopulation might be more sophisticated than thought before.

\section{Granulocytes}

Three different granulocyte subpopulations, classical $\mathrm{CD} 16{ }^{+} \mathrm{CD}_{62} \mathrm{~L}^{+}$, anti-inflammatory $\mathrm{CD} 16^{+} \mathrm{CD}_{2} 2 \mathrm{~L}^{-}$and proinflammatory $\mathrm{CD} 16^{\mathrm{dim}} \mathrm{CD} 62 \mathrm{~L}^{+}$, were identified in adult blood [8] (Fig. 1a-c). In neonates, this study is the first to show that classical granulocytes are downregulated, while proinflammatory granulocytes are enhanced in neonates compared with adults.

Percentage of TLR-2 was enhanced on all granulocytes and their subpopulations while the TLR-2 expression was only reduced on proinflammatory granulocytes (Fig. 2e-g). TLR-2 recognizes a large number of ligands, especially on gram-positive bacteria [17, 18]. Apart from the wellcharacterized TLR-2-mediated inflammation, data exist supporting the notion that TLR-2 signalling can lead to the production of the anti-inflammatory cytokine Interleukin $10[19,20]$. Therefore, differently regulated TLR-2 might be partly responsible for the diminished inflammatory neonatal immune response. Of note, expression of soluble factors in cord blood impairs TLR-4-mediated IL-12p70 production and enhances TLR-4-mediated IL-10 production over the first weeks of life [21].

Although percentages of CD11b and CD32 were downregulated on granulocytes in general, we could not detect these alterations in granulocyte subpopulations (Table 1). The reduction of CD11b in neonates is in line 


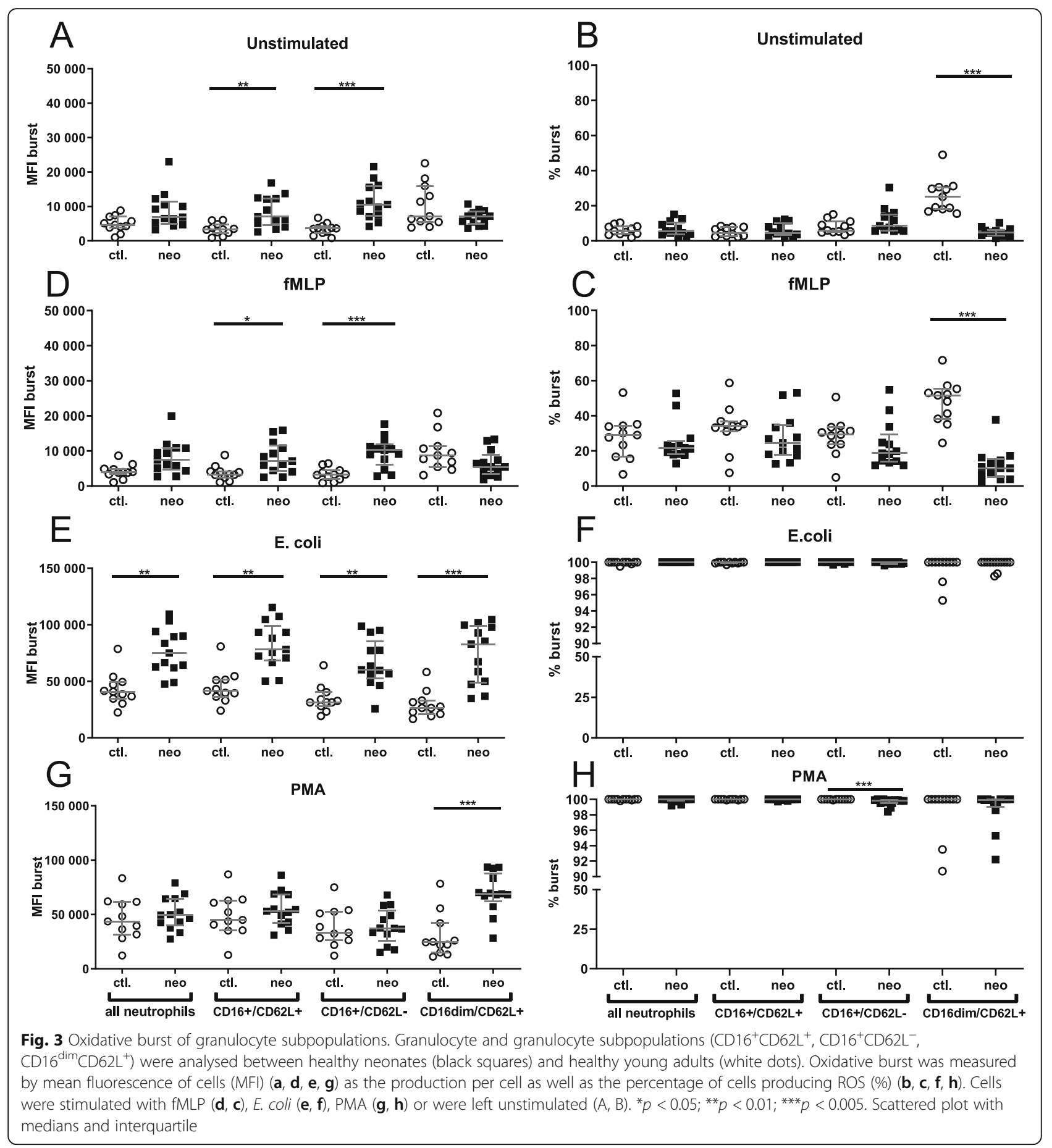

with the finding that a downregulation of CR3 complexes (CD11b/CD18) leads to an impaired recognition of gramnegative bacteria dependant on gestational age [12].

Our results indicate that neutrophils of term infants are partly diminished in their ROS production, especially in proinflammatory granulocytes (Fig. $4 a-f$ ). The reduced ROS production is in line with Usmani et al. [13]. Gessler et al. measured no difference in the percentage of neutrophils undergoing respiratory burst after being stimulated by fMLP or E. coli [14]. Similarly, we did not observe any alterations for granulocytes in general stimulated by fMLP and E. coli in ROS production. Nevertheless, granulocyte subpopulations showed an enhanced ROS production per cell especially after $E$. coli stimulation. Also Shigeoka et al. reported an enhanced ROS response, especially in stressed neutrophils [16]. The 


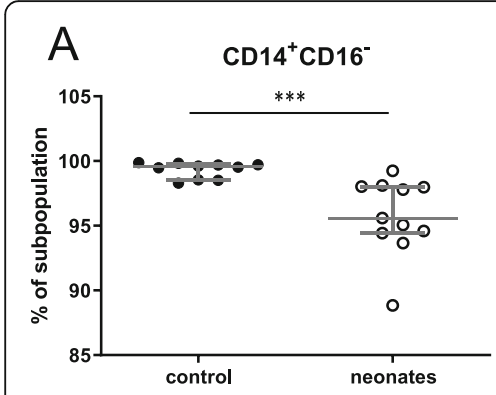

D

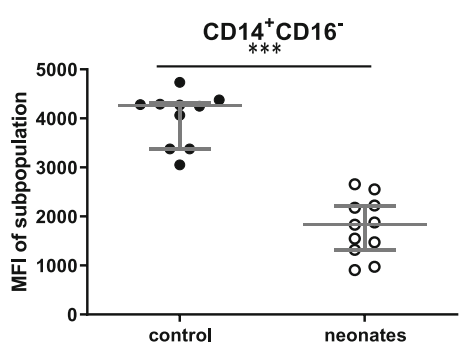

B

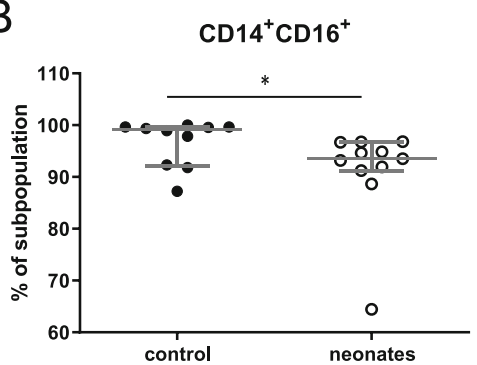

$E$

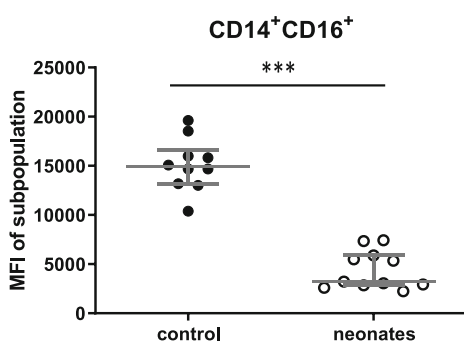

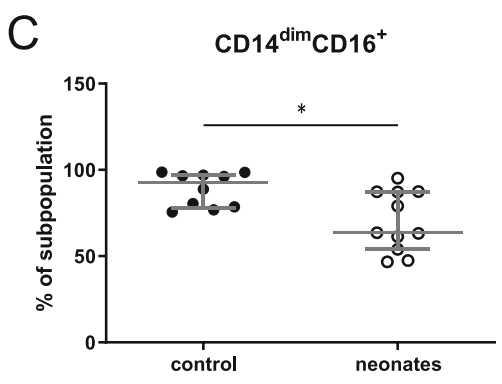

$\mathrm{F}$

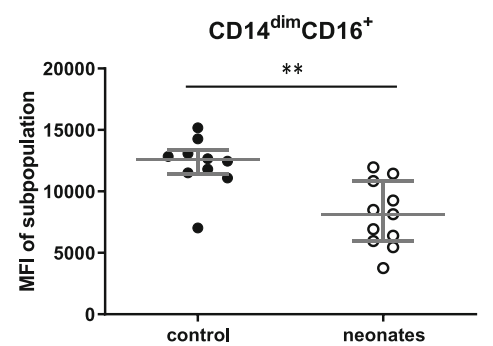

Fig. 4 HLA-DR as activation marker on monocytes and monocyte subpopulations. Neonates (white dots) were compared with healthy controls (black dots). Percentage (\%) (a-c) HLA-DR on the surface of cells and expression of HLA-DR as mean fluorescence intensity (MFI) (D-F) is shown for different monocyte subset. In (a) and (d) anti-inflammatory $\left(\mathrm{CD} 14^{+} \mathrm{CD} 16^{+}\right)$; in (b) and (e) classical monocytes $\left(\mathrm{CD} 14^{+} \mathrm{CD} 16^{-}\right)$and in $(\mathbf{c})$ and $(\mathbf{f})$ inflammatory granulocytes proinflammatory monocytes $\left(\mathrm{CD} 14^{\mathrm{dim}} \mathrm{CD} 16^{+}\right)$are shown. ${ }^{*} p<0.05 ;{ }^{* *} p<0.01 ;{ }^{* * *} p<0.005$. Scattered plot with medians and interquartile

different findings within these studies might be due to divergent experimental setups in choice of stimulants and ROS production readouts. In our study DHR conversion by superoxide radicals into rhodamine is quantified [22]. These radicals are generated by NADPH oxidase which quantitatively differs between neonates and adults [23]. The enhanced NAPDH oxidase activity per cell in contrast to the diminished ROS response of proinflammatory granulocytes might be an indicator of balance between neonatal deficits on the one hand and intact immune responses to fight pathogens effectively on the other hand.

\section{Monocytes}

Our data showed an enhanced percentage of antiinflammatory monocytes while classical monocytes were decreased compared with adults (Fig. 1e-g). Others did not find those differences [24-26]. This might be due to the different classification of monocyte subpopulations since Sohlberg et al. as well as Murphy et al. only distinguished into two major subpopulations, $\mathrm{CD} 14^{++} \mathrm{CD} 16^{-}$ and $\mathrm{CD} 14^{+} \mathrm{CD} 16^{+}$cells. Wisgrill et al. analysed counts of cells while our data compare subpopulation percentages. Especially the $\mathrm{CD} 14^{+} \mathrm{CD} 16^{+}$monocytes have antiinflammatory properties by the secretion of Interleukin 10 [27]. In addition to the lower frequencies of classical monocytes which are confirmed by the study of Sharma et al. in our cohort, these alterations might protect the host from overshooting proinflammatory immune responses facing invading pathogens [28]. In contrast to Sharma et al. we show an increase of anti-inflammatory monocytes without an alteration of inflammatory monocytes, which might be due to differences in gestational age and number of analysed subjects.

We found reduced monocytic HLA-DR expression resembling result by Nguyen et al. and Wisgrill et al. [25, 29]. Our study can expand this knowledge, since our data show a reduced percentage of HLA-DR positive cells in all monocyte subpopulations (Fig. 4a-f). Especially the reduction of CD62L on classical and anti-inflammatory monocytes seems to be a unique neonatal finding in our data since data from adult blood monocytes show CD62L expression especially on these both cell subpopulations but not in proinflammatory monocytes (Table 1) [30,31].

\section{Limitations}

This study only considers the production of ROS in vitro, but not its effectiveness in the killing of pathogens. E. coli as bacterial stimulus of ROS production has been described to play a role in the induction of Escherichia coli early-onset sepsis [32] as an important cause of mortality and morbidity in neonates. However our data cannot specify whether E. coli-induced ROS productions influence the clinical outcome of children. Also, only a limited amount of stimuli could be applied. Since Droussou et al. show an impaired respiratory burst in neonates 
challenged by sepsis, clinical or experimental 'stress' [33], the exact connection between differently regulated monocytes and granulocyte subpopulation, their activation state and the risk of infection still has to be revealed.

Our experiments analysed relative changes of immune cell subpopulations in neonates in comparison with healthy adults, but not absolute counts. Since we cannot provide any tendency for the influence of gestational age, birth weight and the incidence of infection, larger cohort particularly including preterm infants is needed.

\section{Conclusion}

Although granulocyte and monocytes have been characterized by other marker (like CD66b, CD54 or CD49d), our study has demonstrated for the first time that subpopulations of granulocytes and monocytes can be defined by CD14, CD16 and CD62L in neonates. Furthermore, these subpopulations are differently regulated in term neonates compared with adults. Enhanced anti-inflammatory monocytes combined with reduced classical monocytes and classical granulocytes might protect hosts from overshooting immune responses. Although the neonatal is different from the adult immune regulation, our findings show that alterations do not include clear immune deficits. Therefore, it would be conceivable that overwhelming immune responses as well as a higher susceptibility towards infections are limited in neonates. Our data indicate that granulocyte and monocyte subsets, production of ROS as well as the regulation of activation marker are part of a balanced immune system in early life. Whether these balanced immune alterations increase the susceptibility of infections in preterm infants should be object of future studies.

\section{Supplementary information}

Supplementary information accompanies this paper at https://doi.org/10. 1186/s40348-019-0092-y

Additional file 1: Table S1. Characteristics of analysed populations for activation and burst measurements in comparison to healthy young adults. Values presented as mean \pm SD

Additional file 2: Figure S1. A/B Gating strategy for granulocyte and monocyte subpopulations and their activation marker: Representative probe of a new-born infant for activation marker on granulocyte and monocyte subpopulation. After single cell gating and determination of living cells by ZOMBIE, cells were gated by SSC-A and subpopulation marker (CD14, CD16, CD62L) in their subpopulation according to FMOs. CD14 ${ }^{\mathrm{dim}}$ monocytes and CD16 ${ }^{\mathrm{dim}}$ neutrophil population was distinguished by gating the 25th percentile of main neutrophil population.

Additional file 3: Figure S2. Gating strategy for oxidative burst setup. Representative probe of a new-born infant to measure oxidative burst. To clearly distinguish monocyte and granulocyte subpopulations FMOs for anti-CD14, -CD16 and -CD62L gating was used. Subsets were defined as already published by Pillay et al. (2012). CD16 ${ }^{\text {dim }}$ neutrophil population was distinguished by gating the 25 th percentile of main neutrophil population

\section{Abbreviations}

DHR: Dihydrorhodamine 123; EDTA: Ethylene diamine tetraacetic acid; fMLP: N-formyl-methionyl-leucyl-phenylalanine; FMO: Fluorescence minus one; MFI: Mean fluorescence intensity; PFA: Paraformaldehyde; PMA: Phorbol 12-myristate 13-acetate; ROS: Reactive oxygen species; TLR: Toll-like receptor

\section{Acknowledgements}

We thank the patients and their next of kin for participating in this study and paediatric physicians for their support in the recruitment of patients.

\section{Authors' contributions}

JR, MH and AV substantially contributed to the conception and design of the study. IH and FN acquired the data and technically analysed them. JR, MH and AV interpreted the data. $I H, F N$ and JR drafted the manuscript. AL and AV revised it critically for important intellectual content. All authors have read and approved the final manuscript.

\section{Funding}

AV received funding from the University of Greifswald - Käthe-Kluth-ResearchGroup. JR and FN were funded by the Gerhard-Domagk-Program of the University Medicine Greifswald. The funders had no role in the study design, data collection and analysis, decision to publish or preparation of the article.

\section{Availability of data and materials}

The datasets acquired during and/or analysed during the current study are available from the corresponding author upon reasonable request.

\section{Ethics approval and consent to participate}

The study protocol was approved by the ethics committee of the Medical Faculty, University of Greifswald (registration code B133/12). All parents and adult donors provided written informed consent.

\section{Consent for publication}

Not applicable.

\section{Competing interests}

The authors declare that they have no competing interests.

\section{Author details}

${ }^{1}$ Department of Neonatology and Pediatric Intensive Care, University Medicine Greifswald, Greifswald, Germany. ${ }^{2}$ Department of Neurology, University Medicine Greifswald, F.-Sauerbruch-Str., 17475 Greifswald, Germany.

Received: 9 July 2019 Accepted: 24 October 2019 Published online: 28 November 2019

\section{References}

1. Adkins B, Leclerc C, Marshall-Clarke S (2004) Neonatal adaptive immunity comes of age. Nat Rev Immunol 4:553-564

2. Melvan JN, Bagby GJ, Welsh DA, Nelson S, Zhang P (2010) Neonatal sepsis and neutrophil insufficiencies. Int Rev Immunol 29:315-348

3. Lipp $P$ et al (2017) Less neutrophil extracellular trap formation in term newborns than in adults. Neonatology 111:182-188

4. Genel F, Atlihan F, Ozsu E, Ozbek E (2010) Monocyte HLA-DR expression as predictor of poor outcome in neonates with late onset neonatal sepsis. J Infect 60:224-228

5. Dreschers S et al (2016) Reduced PICD in monocytes mounts altered neonate immune response to Candida albicans. PLoS One 11:e0166648

6. Brook B, Harbeson D, Ben-Othman R, Viemann D, Kollmann TR (2017) Newborn susceptibility to infection vs. disease depends on complex in vivo interactions of host and pathogen. Semin Immunopathol 39:615-625

7. Sugitharini V, Prema A, Berla Thangam E (2013) Inflammatory mediators of systemic inflammation in neonatal sepsis. Inflamm Res 62:1025-1034

8. Pillay J et al (2012) A subset of neutrophils in human systemic inflammation inhibits T cell responses through Mac-1. J Clin Invest 122:327-336

9. Pillay J et al (2010) Functional heterogeneity and differential priming of circulating neutrophils in human experimental endotoxemia. J Leukoc Biol 88:211-220

10. Cortjens B et al (2017) Neutrophil subset responses in infants with severe viral respiratory infection. Clin Immunol (Orlando, Fla.) 176:100-106

11. Masuda M, Roos D (1993) Association of all three types of Fc gamma R (CD64, CD32, and CD16) with a gamma-chain homodimer in cultured human monocytes. J Immunol 151:7188-7195 
12. McEvoy LT, Zakem-Cloud H, Tosi MF (1996) Total cell content of CR3 (CD11b/CD18) and LFA-1 (CD11a/CD18) in neonatal neutrophils: relationship to gestational age. Blood 87:3929-3933

13. Usmani SS, Schlessel JS, Sia CG, Kamran S, Orner SD (1991)

Polymorphonuclear leukocyte function in the preterm neonate: effect of chronologic age. Pediatrics 87:675-679

14. Gessler P, Nebe T, Birle A, Haas N, Kachel W (1996) Neutrophil respiratory burst in term and preterm neonates without signs of infection and in those with increased levels of C-reactive protein. Pediatr Res 39:843

15. Kallman J, Schollin J, Schalen C, Erlandsson A, Kihlstrom E (1998) Impaired phagocytosis and opsonisation towards group B streptococci in preterm neonates. Arch Dis Child Fetal Neonatal Ed 78:F46-F50

16. Shigeoka AO, Charette RP, Wyman ML, Hill HR (1981) Defective oxidative metabolic responses of neutrophils from stressed neonates. J Pediatr 98: 392-398

17. Yoshimura A et al (1999) Cutting edge: recognition of Gram-positive bacterial cell wall components by the innate immune system occurs via Toll-like receptor 2. J Immunol (Baltimore, Md. : 1950) 163:1-5

18. Sabroe I et al (2003) Selective roles for Toll-like receptor (TLR)2 and TLR4 in the regulation of neutrophil activation and life span. J Immunol (Baltimore, Md.: 1950) 170:5268-5275

19. Andrade EB et al (2013) TLR2-induced IL-10 production impairs neutrophil recruitment to infected tissues during neonatal bacterial sepsis. J Immunol (Baltimore, Md.: 1950) 191:4759-4768

20. Dillon $\mathrm{S}$ et al (2004) A Toll-like receptor 2 ligand stimulates Th2 responses in vivo, via induction of extracellular signal-regulated kinase mitogenactivated protein kinase and c-Fos in dendritic cells. J Immunol (Baltimore, Md.: 1950) 172:4733-4743

21. Belderbos ME et al (2012) Breastfeeding modulates neonatal innate immune responses: a prospective birth cohort study. Pediatr Allergy Immunol 23:65-74

22. HENDERSON LM, CHAPPELL JB (1993) Dihydrorhodamine 123: a fluorescent probe for superoxide generation? Eur J Biochem 217:973-980

23. Ambruso DR, Stork LC, Gibson BE, Thurman GW (1987) Increased activity of the respiratory burst in cord blood neutrophils: kinetics of the NADPH oxidase enzyme system in subcellular fractions. Pediatr Res 21:205-210

24. Sohlberg E, Saghafian-Hedengren S, Bremme K, Sverremark-Ekström E (2011) Cord blood monocyte subsets are similar to adult and show potent peptidoglycan-stimulated cytokine responses. Immunology 133:41-50

25. Wisgrill $L$ et al (2016) Reduced TNF-alpha response in preterm neonates is associated with impaired nonclassic monocyte function. J Leukoc Biol 100: 607-612

26. Murphy FJ, Reen DJ (1996) Differential expression of function-related antigens on newborn and adult monocyte subpopulations. Immunology 89: 587-591

27. Skrzeczynska-Moncznik J et al (2008) Peripheral blood CD14high CD16+ monocytes are main producers of IL-10. Scand J Immunol 67:152-159

28. Sharma AA et al (2015) Impaired NLRP3 inflammasome activity during fetal development regulates IL-1 beta production in human monocytes. Eur J Immunol 45:238-249

29. Nguyen $\mathrm{M}$ et al (2010) Acquisition of adult-like TLR4 and TLR9 responses during the first year of life. PLoS One 5:e10407

30. Wong KL et al (2012) The three human monocyte subsets: implications for health and disease. Immunol Res 53:41-57

31. Ingersoll MA et al (2010) Comparison of gene expression profiles between human and mouse monocyte subsets. Blood 115:e10-e19

32. Mendoza-Palomar N et al (2017) Escherichia coli early-onset sepsis: trends over two decades. Eur J Pediatr 176:1227-1234

33. Drossou V et al (1997) Impact of prematurity, stress and sepsis on the neutrophil respiratory burst activity of neonates. Biol Neonate 72:201-209

\section{Publisher's Note}

Springer Nature remains neutral with regard to jurisdictional claims in published maps and institutional affiliations.

\section{Submit your manuscript to a SpringerOpen ${ }^{\circ}$ journal and benefit from:}

- Convenient online submission

- Rigorous peer review

- Open access: articles freely available online

- High visibility within the field

- Retaining the copyright to your article

Submit your next manuscript at $\boldsymbol{\nabla}$ springeropen.com 